\title{
TRAIL and cancer immunotherapy: Take a walk on the short side
}

Diego de Miguel $^{1}$ and Julian Pardo ${ }^{1,2,3,4, *}$

${ }^{1}$ Fundación Instituto de Investigación Sanitaria Aragón (IIS Aragón), Biomedical Research

Centre of Aragón (CIBA), 50009, Zaragoza

${ }^{2}$ Aragon I+D Foundation (ARAID). Zaragoza. Spain.

${ }^{3}$ Department Biochemistry and Molecular and Cell Biology and Dept. Microbiology, Preventive Medicine and Public Health, University of Zaragoza. 50009, Zaragoza, Spain.

${ }^{4}$ Centro de Investigación Biomédica en Red de Bioingeniería, Biomateriales y Nanomedicina (CIBER-BBN), 50018 Madrid, Spain.

*Corresponding Author: Julián Pardo, Biomedical Research Centre of Aragon (CIBA), Avda. San Juan Bosco 13, 50009, Zaragoza, Spain; e-mail: pardojim@unizar.es; phone: +34876554338.

The authors have no conflict of interest

Running title: TRAILshort, a novel cancer immunoevasion mechanism

Funding: DdM is supported by a Sara Borrell contract from Health Institute Carlos III. Work at the JP lab is supported by ARAID, FEDER, Aragon Government (B29_17R), Ministry of Science, Innovation and Universities (SAF2017-83120-C2-1-R), ASPANOA, Carrera de la Mujer de Monzón, Fundacion Inocente Inocente and Health Institute Carlos III. 


\section{Abstract}

Recent work shows that TRAILshort, a membrane-bound short form of TRAIL, is expressed by human cancer cells and protects them from TRAIL-induced cell death. A monoclonal antibody that selectively targets TRAILshort enhances cancer susceptibility to TRAIL and increases the efficacy of autologous CD8+T cells in ex vivo primary tumours. 


\section{Main text}

In this issue of Clinical Cancer Research, Aboulnasr and colleagues (1), in an elegant well controlled study, provide new experimental evidences that may partly explain the intrinsic resistance of cancer cells to TRAIL and the low efficacy of TRAIL in cancer treatment: a great proportion of human cancers express and secrete a shorter form of TRAIL, TRAILshort, that does not show cytotoxic potential but competes with the binding of TRAIL to its two receptors TRAIL-R1 and R2, impeding the activation of TRAIL-induced cell death pathways in cancer cells.

TRAIL activates cell death and/or survival pathways upon binding to two specific receptors in humans TRAIL-R1/DR4) and TRAIL-R2/DR5. In addition, it possesses two non-functional decoy receptors, DcR1 and DcR2. The ability to induce cell death together with the low toxicity against healthy tissue/cells aimed several clinical trials to test its efficacy against different cancers alone or in combination with other molecules. However, the original promising results employing animal models (2) were not translated into human patients and the results of the clinical trials were disappointing as they did not reveal any significant benefit of TRAIL in disease progression and outcome (3). Indeed, different studies showed that most cancer cell lines as well as primary patient-derived cells were resistant to TRAIL in vitro. In addition, the contribution of TRAIL to CD8+ cytotoxic T cell-mediated cell death is not clear yet.

Aboulnasr et al identify RNA transcripts for TRAILshort in 40\% of human cancers in two data bases (Gene Expression Omnibus (GEO) and the Atlas of Human Cancer), of which $15 \%$ expressed high/very high levels. These results were subsequently validated at the protein level by IHC in tissues from different cancer types and its functional relevance tested in vitro and in vivo employing a new humanized antibody that selectively binds TRAILshort but not the long active form of TRAIL. The authors found that several human cancer cell lines from different origin as well as primary lymphoma cells were more sensitive to TRAIL-induced apoptosis in the presence of the antiTRAILshort antibody in vitro. A similar result was also found in xenograft mouse models (NSG) in vivo employing two hematological cell lines, albeit in this case the results were modest. Meritoriously, authors employ an ex vivo autologous human model of lymphoma to show that anti-TRAILshort antibody enhances autologous $\mathrm{CD} 8^{+} \mathrm{T}$ cellsmediated elimination of lymphoma cells, suggesting that TRAIL is used by CD8 ${ }^{+} \mathrm{T}$ cells to kill cancer cells, a question that is not clear yet.

TRAILshort, firstly identified in HIV-infected patients produced by alternative splicing, lacks exons 3 and 4 resulting in the appearance of an unique C-terminal 11 aminoacid sequence followed by a premature stop codon in exon 5 (4). TRAILshort lacks the typical TNF-homology domain, which causes the loss of the Cysteine 230, which is responsible for stabilizing the trimeric conformation of full-length TRAIL, impeding the homo-trimerisation of TRAILshort and accounting for its monomeric nature. Interestingly, in contrast to full-length TRAIL that can also be found in a soluble form 
due to cleavage by a putative metalloprotease, TRAILshort could only be detected in a non-cleaved form and associated with microvesicles/exosomes. Noteworthy, full length TRAIL was also shown to be released associated to exosomes by CD8+T cells (3), which implies that TRAILshort, in addition to blocking soluble TRAIL and antiTRAIL-R agonistic antibodies, is also able to block the exosome-bound full length TRAIL. Regarding tumour spatial heterogeneity, expression and secretion of TRAILshort in exosomes by a few number of cancer cell clones in specific tumour sites might benefit the expansion of distal clones that do not express it, promoting a community immunoevasion mechanism. These are important questions for designing future clinical trials combining immune checkpoint inhibitors (ICIs) with antiTRAILshort antibodies.

Expression of TRAILshort provides an additional explanation to the low efficacy of TRAIL in the elimination of cancer cells in vitro and in vivo, and to the disappointing clinical trial results using TRAIL or agonistic anti-TRAIL-R1/2 antibodies. Importantly, only those cancer types that express TRAILshort respond to the anti-TRAILshort antibody, reinforcing the validity of the conclusions and advancing a potential biomarker to predict those tumors that will most benefit of this potential therapy. Besides inducing cell death, TRAIL can also activate proliferative/pro-survival pathways such as NF-kB. Thus, it will be important to study if TRAILshort, in contrast to cell death activation, can still activate these pathways. If so, blocking TRAILshort would not only enhance susceptibility to TRAIL-induced cell death but, in addition, would directly impact tumour proliferation/survival.

Previous studies have shown a role for the TRAIL pathway in cancer immunosurveillance (5). Intriguingly, the contribution of TRAIL to the elimination of cancer cells by mature NK or cytotoxic CD8+ T cells is very low in comparison with other effector molecules like perforin and granzymes or even Fas Ligand. This low activity has been attributed to the expression of intracellular molecules that regulate the extrinsic apoptotic pathway like FLIP, members of the IAP family or even the decoy receptors DcR1 and DcR2. However, the relevance of all of them for TRAIL immunosurveillance and immunotherapy is unclear. Now, TRAILshort emerges as a novel contributor to cancer immunoevasion and a potential target to enhance TRAIL antitumoral effect (Figure). This finding suggests that TRAILshort blockade might synergize with granule exocytosis (perforin and granzymes) to enhance cancer cell killing, albeit this hypothesis will require further experimental validation. Blocking TRAILshort enhances the anti-tumoral action of $\mathrm{CD} 8+\mathrm{T}$ cells, which prompts us to speculate on a potential synergy with ICIs against PD1/L1, TIM3 and/or LAG3. It will also be interesting to analyse the effect of anti-TRAILshort antibodies on NK cell mediated-immunosurveillance and immunotherapy (i.e. by combining anti-TRAILshort with antibodies against NK cell checkpoints like NKG2A or with antibodies with ADCC activity) since TRAIL is also a component of the cytotoxic machinery of NK cells. 
Expression of TRAILshort seems to be highly dependent on activation of certain proinflammatory pathways, such as Type I interferon and TLR activation. In this line, in contrast to HIV patients, where both infected and non-infected cells expressed TRAILshort and were protected from TRAIL-induced cell death (4), expression of TRAILshort in cancer patients was exclusively found in cancer cells. Thus, selective activation of certain inflammatory signaling pathways in cancer cells would lead to TRAILshort expression and acquired TRAIL-resistance. This hypothesis will require further experimental validation in future studies to better understand the specific settings where TRAILshort might be expressed and design more efficient protocols employing anti-TRAILshort antibodies. The preliminary data presented by Aboulnasr and collaborators show that blocking of TRAILshort did not increase the sensitivity of non-activated PBLs to TRAIL. Activated PBLs were not included in the analyses, which might be important considering the role of type I IFN in the regulation of TRAILshort expression. TRAIL has shown a very good safety profile in several studies and clinical trials, with very few adverse effects (3). Nonetheless, in light of the results presented here, the potential toxicity (including immune-related adverse effects) of antiTRAILshort antibodies alone or in combination with TRAIL receptor agonists will require careful analyses in animal models before clinical trials employing these combinations can be proposed.

The discovery of a new mechanism/function is always exciting, especially when it helps to explain previous negative results of molecules already tested at the clinical level, providing new ways to improve their efficacy. Obviously, it raises many new questions aimed at better understanding the biological significance of the new findings and the opportunities to use this knowledge for the clinical benefit of cancer patients. Thus, the present study by Andrew Badley's lab opens a lot of questions, but most importantly it contributes to understand the role of TRAIL during the elimination of cancer cells by effector CD8+T cells, and provides a potential new target to increase the efficacy of TRAIL-based treatments for cancer. 


\section{References}

1. Aboulnasr F, Krogman A, Graham RP, Cummins NW, Misra A, Garcia-Rivera E, et al. Human cancers express TRAILshort, a dominant negative TRAIL splice variant, which impairs immune effector cell killing of tumor cells. Clinical Cancer Research 2020.

2. Walczak H, Miller RE, Ariail K, Gliniak B, Griffith TS, Kubin M, et al. Tumoricidal activity of tumor necrosis factor-related apoptosis-inducing ligand in vivo. Nat Med 1999;5(2):157-63.

3. de Miguel D, Lemke J, Anel A, Walczak H, Martinez-Lostao L. Onto better TRAILs for cancer treatment. Cell Death Differ 2016;4(10):174.

4. Nie Z, Aboulnasr F, Natesampillai S, Burke SP, Krogman A, Bren GD, et al. Both HIV-Infected and Uninfected Cells Express TRAILshort, Which Confers TRAIL Resistance upon Bystander Cells within the Microenvironment. J Immunol 2018;200(3):1110-23.

5. $\quad$ Martinez-Lostao L, Anel A, Pardo J. How Do Cytotoxic Lymphocytes Kill Cancer Cells? Clin Cancer Res 2015;21(22):5047-56 doi 10.1158/10780432.CCR-15-0685. 


\section{Figure legend}

TRAILshort counteracts cell death induced by TRAIL. Tumour cells express a short form of TRAIL, TRAILshort that is secreted bound to exosomes. TRAILshort binds to TRAIL-R1 and 2, DR4 and DR5, but does not show cytotoxic potential. Contrary it competes with the binding of TRAIL to DR4 and DR5, impeding the activation of cell death pathways in cancer cells by TRAIL-expressing cytotoxic CD8+T cell or by treatments like recombinant TRAIL or agonistic anti-DR antibodies. A monoclonal antibody that selectively binds to TRAILshort prevents binding of TRAILshort to DR4 and DR5 and enhances the susceptibility of cancer cells to TRAIL-expressing cytotoxic CD8+T cells or TRAIL-based treatments. 


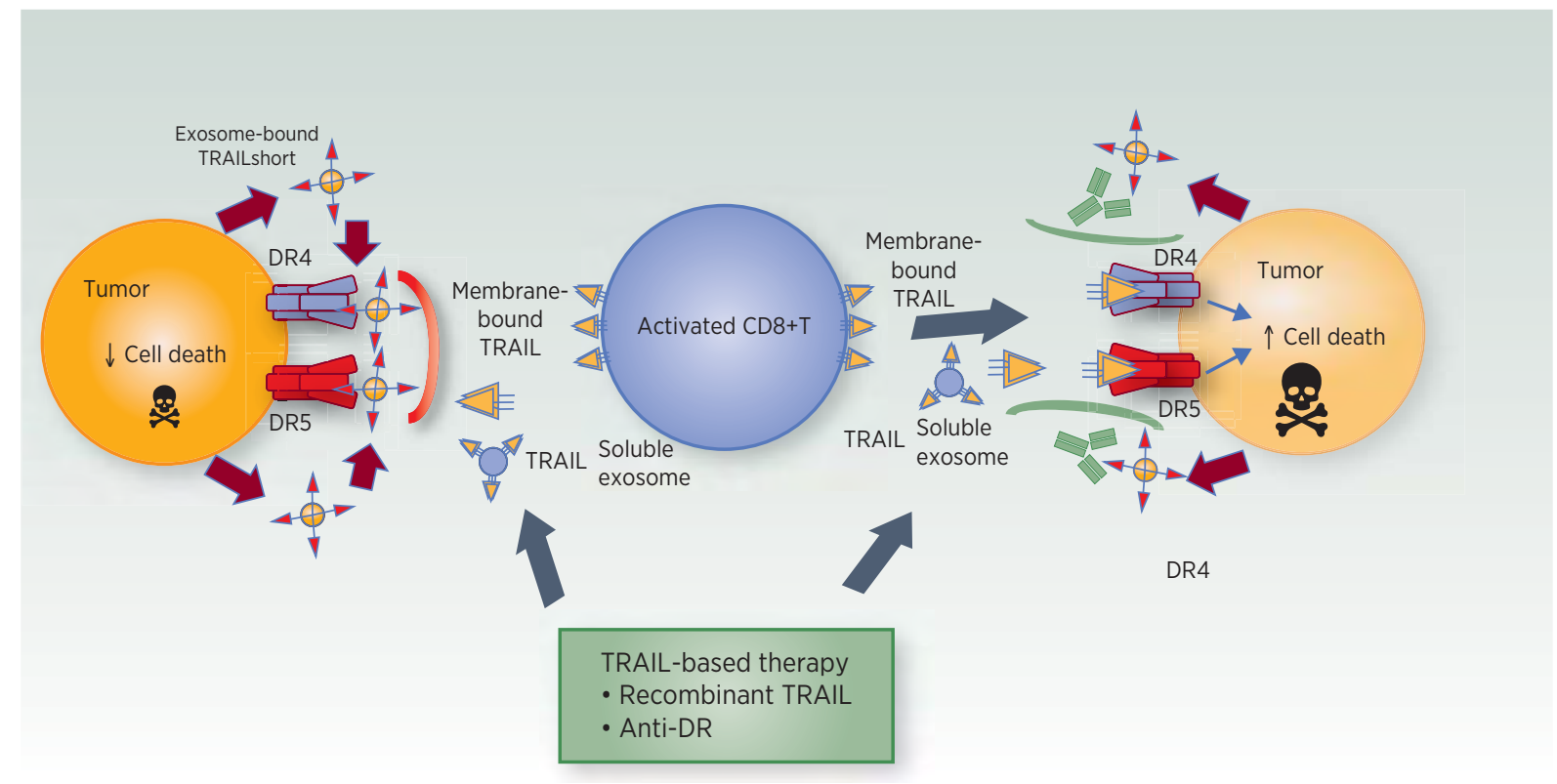

Downloaded from https://bloodcancerdiscov.aacrjournals.org at Edward G. Miner Library, UNIV OF ROCHESTER on August 28, 2020. Copyright 2020 American Association for Cancer Research. 